\title{
News dal XVII Convegno del Gruppo di Studio di Dialisi Peritoneale
}

\author{
Roberto Russo
}

\author{
U.O. di Nefrologia Universitaria, Azienda Universitaria Consorziale Policlinico, Bari
}

\begin{abstract}
NEWS FROM THE XVII CONFERENCE OF THE STUDY GROUP ON PERITONEAL DIALYSIS
Abstract. The XVII conference of the Study Group on Peritoneal Dialysis held in Montecatini has just ended. A large number of participants followed the scientific sessions inspired by the convention motto: "peritoneal dialysis: more information and better quality of care."
\end{abstract}

Key words: Peritoneal dialysis, CAPD, Convention

Conflict of interest: None.

Financial support: None.

Accettato: 9 Aprile 2014

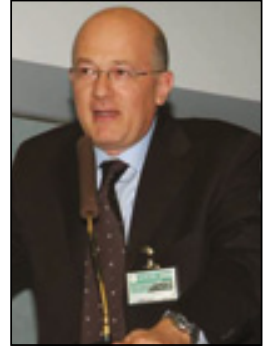

Inaugurato dal Presidente della Società Italiana di Nefrologia, si è svolto a Montecatini il XVII Convegno del Gruppo di Studio di Dialisi Peritoneale (GdSDP) (Fig. 1). Il successo dell'iniziativa è testimoniato dal numero di partecipanti: 360 nefrologi, 80 specializzandi e infermieri, 95 dipendenti di aziende farmaceutiche e 20 aziende sponsor dell'evento.

Roberto Russo Il motto del Convegno è stato: "dialisi peritoneale: più cultura e migliore qualità delle cure". "Più cultura" perché ancora oggi, secondo il Comitato Scientifico del Convegno, non è abbastanza diffusa la cultura della dialisi domiciliare. A questo tema è stata dedicata la tavola rotonda di apertura del Convegno, che ha visto, tra l'altro, la partecipazione dei pazienti nella persona della Presidente dell'ANED, la Dr.ssa Valentina Paris. Nel corso di essa, si è discusso di modelli organizzativi, di terapia nutrizionale, di trapianto preemptive e di emodialisi domiciliare. Quest'ultima opzione terapeutica dell'uremia può rappresentare il naturale proseguimento della terapia dialitica in quei pazienti in dialisi peritoneale che devono essere trasferiti all'emodialisi; si tratta di una cultura della domiciliarità da diffondere anche al di fuori dei luoghi per addetti ai lavori, così come è stato fatto dal Gruppo Toscano di Dialisi Peritoneale, che ha avviato il progetto "conoscere per educare, educare per conoscere". Testimoni dell'iniziativa una classe di ragazzi della Scuola Media di San Miniato che, insieme ai loro insegnanti, hanno partecipato al Convegno presentando materiale didattico e divulgativo sul tema della conoscenza e della prevenzione della malattia renale.

Altra mission del Convegno è stata quella di implementare una "migliore qualità delle cure". A ciò sono stati dedicati una tavola rotonda in cui sono state discusse, dai coordinatori, le otto Best Practice recentemente prodotte dal GdSDP, un lunch seminar sulle procedure di audit e di individuazione dei "Key Performance Indicators" e le sessioni di PD basic, che hanno visto l'attiva partecipazione dei numerosi specializzandi presenti al Convegno.

Sul tema del miglioramento delle cure si sono tenute una sessione dedicata alla telemedicina, che può trovare interessanti applicazioni nelle terapie domiciliari, una tavola rotonda su trapianto e dialisi peritoneale, in cui sono stati discussi i rapporti tra terapie antirigetto e peritonite sclerosante, e una tavola rotonda sulle soluzioni e sui loro effetti su biocompatibilità e outcome. Interessanti le applicazioni della telemedicina, che consente la trasmissione dei dati clinici e la possibilità di videocall, di teleconsulto e di e-learning su piattaforme informatiche utilizzabili anche per creare una rete territoriale tra paziente, specialista ospedaliero e medico di base.

Il consenso informato è stato discusso in una tavola rotonda che si è conclusa con la proposta di sviluppare un format di consenso da poter utilizzare su tutto il territorio nazionale.

Tra le sessioni più seguite, quella in cui è stato presentato il censimento di dialisi peritoneale 2012, a cui hanno partecipato 224 centri italiani di dialisi peritoneale. Il censimento ha raccolto i dati di 4299 pazienti sulle cause di drop out, sulle infezioni e sulla peritonite sclerosante. Un leggero ottimismo emerge dai dati di prevalenza da cui risulta un aumento dei pazienti del $7.29 \%$ rispetto al 2010 . Si riducono anche il ruolo 


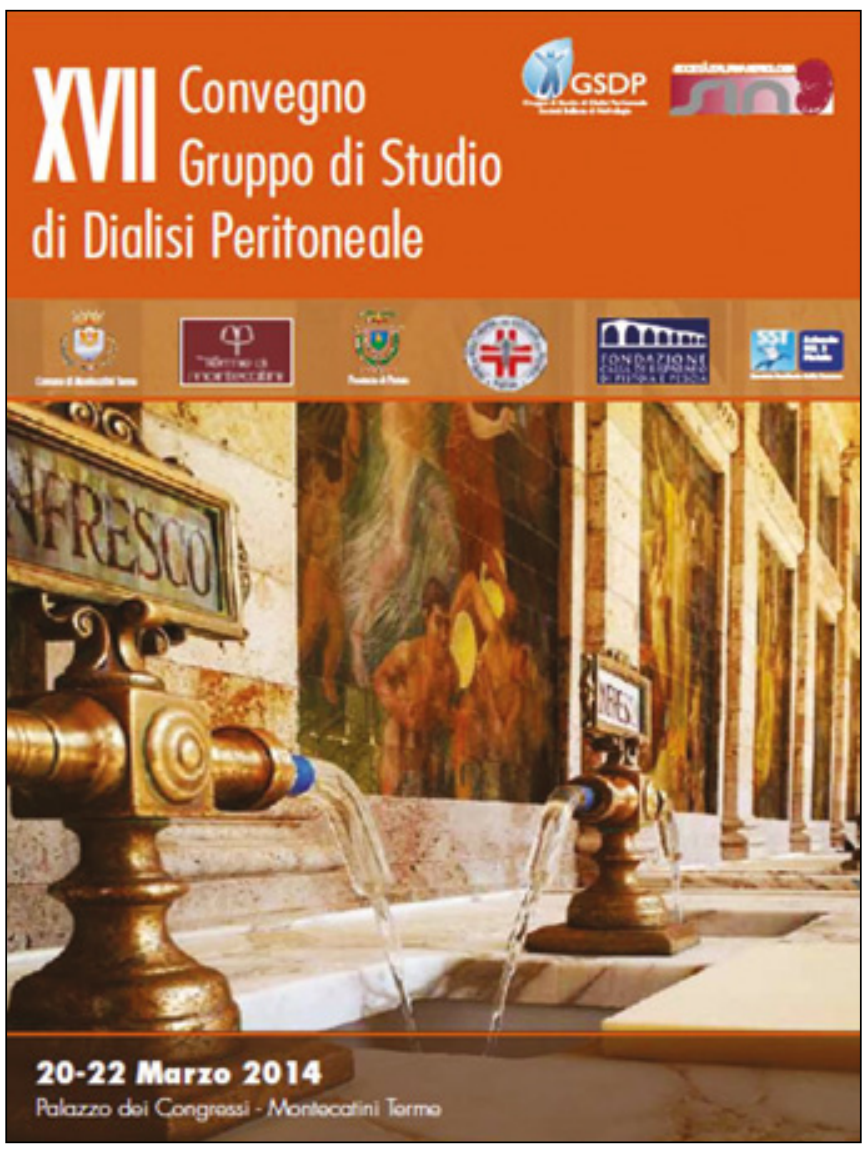

Fig. 1 - Locandina del XVII Convegno del Gruppo di Studio di Dialisi Peritoneale.

della peritonite quale causa di drop out, passando dal $37.9 \%$ al $28.2 \%$, con un'incidenza di $1 / 42.3 \mathrm{mesi} /$ paziente, e il rischio di peritonite sclerosante, con 43 casi registrati, 0.505 episodi/100 anni/paziente. I dati completi sono consultabili sul sito web del GdSDP (www.dialisiperitoneale.org).

Infine, molto spazio è stato dedicato alle comunicazioni orali e ai poster, essendo pervenuti 90 abstract. Un numero in crescita rispetto ai Convegni di Alba e Bari (rispettivamente
75 e 52 abstract $)$.

Il Convegno è stato anche l'occasione per ricordare, con delle iniziative editoriali, due colleghi "peritonealisti" recentemente scomparsi. Ad Amedeo De Vecchi è stato dedicato un numero speciale del Giornale di Tecniche Nefrologiche e Dialitiche, dal titolo "Dialisi peritoneale: dialisi marginale? Opinioni a confronto" e a Roberta Marcon è stata dedicata una raccolta di testimonianze di pazienti e familiari, dal titolo "Voci da dentro. Fotolibro emozionale".

Di contorno alle sessioni scientifiche un concorso fotografico, dal titolo "scatti d'acqua, la vita in un click", che ha visto la partecipazione di numerosi colleghi.

Il Convegno, di tre giorni, si è concluso con un arrivederci a Giulianova nel 2016 per il XVIII Convegno del GdSDP.

\section{Riassunto}

Si è appena concluso a Montecatini il XVII Convegno del Gruppo di Studio di Dialisi Peritoneale. Numerosi i partecipanti che hanno seguito le sessioni scientifiche ispirate al motto: "dialisi peritoneale: più cultura e migliore qualità delle cure".

Parole chiave: Dialisi peritoneale, CAPD, Convegno.

Dichiarazione di conflitto di interessi: L'Autore dichiara di non avere conflitto di interessi.

Contributi economici agli Autori: L'Autore dichiara di non aver ricevuto sponsorizzazioni economiche per la preparazione dell'articolo.

Indirizzo degli Autori:

Dr. Roberto Russo

U.O. di Nefrologia

Azienda Ospedaliero-Universitaria

Consorziale Policlinico di Bari

Piazza G. Cesare 11

70124 Bari

roberto.russo@policlinico.ba.it 